Article

\title{
Study on the Algebraic Structure of Refined Neutrosophic Numbers
}

\author{
Qiaoyan Li ${ }^{1}$, Yingcang Ma ${ }^{1, *}$, Xiaohong Zhang ${ }^{2}$ and Juanjuan Zhang ${ }^{1}$ \\ 1 School of Science, Xi'an Polytechnic University, Xi'an 710048, China \\ 2 School of Arts and Sciences, Shaanxi University of Science \& Technology, Xi'an 710021, China \\ * Correspondence: mayingcang@126.com
}

Received: 28 May 2019; Accepted: 22 July 2019; Published: 27 July 2019

\begin{abstract}
This paper aims to explore the algebra structure of refined neutrosophic numbers. Firstly, the algebra structure of neutrosophic quadruple numbers on a general field is studied. Secondly, The addition operator $\oplus$ and multiplication operator $\otimes$ on refined neutrosophic numbers are proposed and the algebra structure is discussed. We reveal that the set of neutrosophic refined numbers with an additive operation is an abelian group and the set of neutrosophic refined numbers with a multiplication operation is a neutrosophic extended triplet group. Moreover, algorithms for solving the neutral element and opposite elements of each refined neutrosophic number are given.
\end{abstract}

Keywords: neutrosophic extended triplet group; neutrosophic quadruple numbers; refined neutrosophic numbers; refined neutrosophic quadruple numbers; neutrosophic set

\section{Introduction}

The notion of neutrosophic set was proposed by F. Smarandache [1], which is an extension of fuzzy set and in order to solve real-world problems. A neutrosophic set has three membership functions, and each membership degree is a real standard or non-standard subset of the nonstandard unit interval ] $0^{-}, 1^{+}\left[=0^{-} \cup[0,1] \cup 1^{+}\right.$.

In recent years, the idea of neutrosophic set has been applicable in related algebraic structures. Among these algebraic structures, Smarandache and Ali [2] proposed the algebraic system neutrosophic triplet group (NTG), which is an extension of the classical group but the neutral element is different from the classical algebraic unit element. To regard the unit element as a special neutral element, the neutrosophic extended triplet group (NETG) has been proposed [3,4] and the classical group is regarded as a special case of a NETG. Moreover, some research papers have carried out in-depth research based on NTG (NETG). For example, the inclusion relations of neutrosophic sets [5], neutrosophic triplet coset [6], neutrosophic duplet semi-groups [7], generalized neutrosophic extended triplet group [8], AG-neutrosophic extended triplet loops [9,10], the neutrosophic set theory to pseudo-BCI algebras [11], neutrosophic triplet ring and a neutrosophic triplet field [12,13], neutrosophic triplet normed space [14], neutrosophic soft sets [15], neutrosophic vector spaces [16] and so on have been studied.

As an example of NETG, Ma [8] revealed that for each $n \in Z^{+}, n \geq 2,\left(Z_{n}, \otimes\right)$ is a commutative NETG if and only if the factorization of $n$ is a product of single factors. As another example, Ma [17] showed that the set of neutrosophic quadruple numbers with a multiplication operation is a NETG. The concept of neutrosophic numbers of the form $a+b I$, where $I$ is the indeterminacy with $I^{n}=$ $I$, and, $a$ and are real or complex numbers. If $I$ into many types of indeterminacies $I_{1}, I_{2}, \cdots, I_{q}$, in [18], Smarandache extended the neutrosophic numbers $a+b I$ into refined neutrosophic numbers of the form $a+b_{1} I_{1}+b_{2} I_{2}+\cdots+b_{n} I_{n}$, where $a, b_{1}, b_{2}, \cdots, b_{n}$ are real or complex numbers and considered the refined neutrosophic set based on these refined neutrosophic numbers. The notion of 
neutrosophic quadruple number, which has form: $N Q=a+b T+c I+d F$ where $a, b, c, d$ are real (or complex) numbers; and $T$ is the truth/membership/probability; $I$ is the indeterminacy; and $F$ is the false/membership/improbability are called Neutrosophic Quadruple (Real, respectively, Complex) Numbers. " $a$ " is called the known part of $N Q$, while $b T+c I+d F$ is called the unknown part of $N Q$. Similar to refined neutrosophic numbers, if $T$ can be split into many types of truths, $T_{1}, T_{2}, \cdots, T_{p}, I$ into many types of indeterminacies, $I_{1}, I_{2}, \cdots, I_{r}$, and $F$ into many types of falsities, $F_{1}, F_{2}, \cdots, F_{r}$, we can get the refined neutrosophic quadruple numbers. We know that the set of neutrosophic quadruple numbers with a multiplication operation is a NETG. In this paper, we explore the algebra structure of refined neutrosophic numbers (refined neutrosophic quadruple numbers) and give new examples of NETG. In fact, the solving method of the neutral element and opposite elements for each refined neutrosophic number is different from the solving method for each neutrosophic quadruple number.

The paper is organized as follows. Section 2 gives the basic concepts. In Section 3, we show that the set of neutrosophic quadruple numbers on the general field with a multiplication operation also consists of a NETG. In Section 4, the algebra structure of refined neutrosophic numbers and refined neutrosophic quadruple numbers are studied. Finally, the summary and future work is presented in Section 5 .

\section{Basic Concepts}

In this section, we provide the related basic definitions and properties of NETG, neutrosophic quadruple numbers, and refined neutrosophic numbers (for details, see [3,4,18-20]).

Definition $1([3,4])$. Let $N$ be a non-empty set together with a binary operation $*$. Then, $N$ is called a neutrosophic extended triplet set if, for any $a \in N$, there exists a neutral of " $a$ " (denote by neut $(a)$ ), and an opposite of " $a$ "(denote by anti $(a))$, such that neut $(a) \in N$, anti $(a) \in N$ and:

$$
a * \operatorname{neut}(a)=\operatorname{neut}(a) * a=a, \quad a * \operatorname{anti}(a)=\operatorname{anti}(a) * a=\operatorname{neut}(a) .
$$

The triplet $(a, n e u t(a)$, anti $(a))$ is called a neutrosophic extended triplet.

Definition $2([3,4])$. Let $(N, *)$ be a neutrosophic extended triplet set. Then, $N$ is called a neutrosophic extended triplet group (NETG), if the following conditions are satisfied:

(1) $(N, *)$ is well-defined, i.e., for any $a, b \in N$, one has $a * b \in N$.

(2) $(N, *)$ is associative, i.e., $(a * b) * c=a *(b * c)$ for all $a, b, c \in N$.

A NETG $N$ is called a commutative NETG if for all $a, b \in N, a * b=b * a$.

Proposition $1([4]) .(N, *)$ be a NETG. We have:

(1) neut (a) is unique for any $a \in N$.

(2) neut $(a) *$ neut $(a)=\operatorname{neut}(a)$ for any $a \in N$.

(3) neut (neut $(a))=$ neut (a) for any $a \in N$.

Definition $3([18,19])$. A neutrosophic number is a number of the form $(a, b I)$, where $I$ is the indeterminacy with $I^{2}=I$, and $a$ and $b$ are real or complex numbers. A refined neutrosophic number is a number of the form $\left(a_{0}, a_{1} I_{1}, a_{2} I_{2}, \cdots, a_{n} I_{n}\right)$, where $I_{1}, I_{2}, \cdots, I_{n}$ are different types of indeterminacies, and $a_{0}, a_{1}, a_{2}, \cdots, a_{n}$ are real or complex numbers. The set NN defined by

$$
N N=\{(a, b I) \mid a, b \in \mathbb{R} \text { or } \mathbb{C}\} .
$$

is called a neutrosophic set of neutrosophic numbers. The set RNN defined by

$$
R N N=\left\{\left(a_{0}, a I_{1}, a_{2} I_{2}, \cdots, a_{n} I_{n}\right) \mid a_{0}, a_{1}, a_{2}, \cdots, a_{n} \in \mathbb{R} \text { or } \mathbb{C}\right\}
$$


is called a neutrosophic set of refined neutrosophic numbers.

Definition 4 ([18,20]). A neutrosophic quadruple number is a number of the form $(a, b T, c I, d F)$, where $T, I$ and $F$ have their usual neutrosophic logic meanings, i.e., truth, indeterminacy and false, respectively, and $a, b, c, d \in \mathbb{R}$ or $\mathbb{C}$. The set $N Q$ defined by

$$
N Q=\{(a, b T, c I, d F) \mid a, b, c, d \in \mathbb{R} \text { or } \mathbb{C}\} .
$$

is called a neutrosophic set of quadruple numbers. For a neutrosophic quadruple number $(a, b T, c I, d F), a$ is called the known part and $(b T, c I, d F)$ is called the unknown part. The set RNQ defined by

$$
\begin{aligned}
R N Q= & \left\{\left(a, b_{1} T_{1}, b_{2} T_{2}, \cdots, b_{p} T_{p}, c_{1} I_{1}, c_{2} I_{2}, \cdots, c_{q} I_{q}, d_{1} F_{1}, d_{2} F_{2}, \cdots, d_{r} F_{r}\right) \mid\right. \\
& \left.a, b_{1}, b_{2}, \cdots, b_{p}, c_{1}, c_{2}, \cdots, c_{q}, d_{1}, d_{2}, \cdots, d_{r} \in \mathbb{R} \text { or } \mathbb{C}\right\} .
\end{aligned}
$$

is called a neutrosophic set of refined neutrosophic quadruple numbers.

Definition 5 ([18,20]). Let $N$ be a set, endowed with a total order $a \prec b$, named "a prevailed by $b$ ", "a less stronger than $b$ " or "a less preferred than $b$ ". We consider $a \preceq b$ as " $a$ prevailed by or equal to $b$ ", "a less stronger than or equal to $b$ ", or "a less preferred than or equal to $b$ ".

For any elements $a, b \in N$, with $a \preceq b$, one has the absorbance law:

$$
a \cdot b=b \cdot a=\operatorname{absorb}(a, b)=\max (a, b)=b .
$$

which means that the bigger element absorbs the smaller element. Clearly,

$$
a \cdot a=a^{2}=\operatorname{absorb}(a, a)=\max (a, a)=a .
$$

and

$$
a_{1} \cdot a_{2} \cdots a_{n}=\max \left(a_{1}, a_{2}, \cdots, a_{n}\right) .
$$

Analogously, we say that " $a \succ b$ " and we read: "a prevails to $b$ ", " $a$ is stronger than $b$ " or " $a$ is preferred to $b$ ". In addition, $a \succeq b$, and we read: "a prevails or is equal to $b$ ", " $a$ is stronger than or equal to $b$ ", or " $a$ is preferred or equal to $b^{\prime \prime}$.

Definition $6([18,20])$. Consider the set $\{T, I, F\}$. Suppose in an optimistic way we consider the prevalence $\operatorname{order} T \succ I \succ F$. Then, we have: $T I=I T=\max (T, I)=T, T F=F T=\max (T, F)=T, I F=F I=$ $\max (I, F)=I, T T=T^{2}=T, I I=I^{2}=I, F F=F^{2}=F$.

Analogously, suppose in a pessimistic way we consider the prevalence order $T \prec I \prec F$. Then, we have: $T I=I T=\max (T, I)=I, T F=F T=\max (T, F)=F, I F=F I=\max (I, F)=F, T T=T^{2}=T$, $I I=I^{2}=I, F F=F^{2}=F$.

Definition $7([18,20])$. Let $a=\left(a_{1}, a_{2} T, a_{3} I, a_{4} F\right), b=\left(b_{1}, b_{2} T, b_{3} I, b_{4} F\right) \in N Q$. Suppose, in an pessimistic way, the neutrosophic expert considers the prevalence order $T \prec I \prec F$. Then, the multiplication operation is defined as follows:

$$
\begin{aligned}
a * b= & \left(a_{1}, a_{2} T, a_{3} I, a_{4} F\right) *\left(b_{1}, b_{2} T, b_{3} I, b_{4} F\right) \\
= & \left(a_{1} b_{1},\left(a_{1} b_{2}+a_{2} b_{1}+a_{2} b_{2}\right) T,\left(a_{1} b_{3}+a_{2} b_{3}+a_{3} b_{1}+a_{3} b_{2}+a_{3} b_{3}\right) I,\right. \\
& \left.\left(a_{1} b_{4}+a_{2} b_{4}+a_{3} b_{4}+a_{4} b_{1}+a_{4} b_{2}+a_{4} b_{3}+a_{4} b_{4}\right) F\right) .
\end{aligned}
$$

Suppose in an optimistic way the neutrosophic expert considers the prevalence order $T \succ I \succ F$. Then,

$$
\begin{aligned}
a \star b= & \left(a_{1}, a_{2} T, a_{3} I, a_{4} F\right) \star\left(b_{1}, b_{2} T, b_{3} I, b_{4} F\right) \\
= & \left(a_{1} b_{1},\left(a_{1} b_{2}+a_{2} b_{1}+a_{2} b_{2}+a_{3} b_{2}+a_{4} b_{2}+a_{2} b_{3}+a_{2} b_{4}\right) T,\right. \\
& \left.\left(a_{1} b_{3}+a_{3} b_{1}+a_{3} b_{3}+a_{3} b_{4}+a_{4} b_{3}\right) I,\left(a_{1} b_{4}+a_{4} b_{1}+a_{4} b_{4}\right) F\right) .
\end{aligned}
$$


Proposition $2([18,20])$. Let $N Q=\{(a, b T, c I, d F): a, b, c, d \in \mathbb{R}$ or $\mathbb{C}\}$. We have:

(1) $(N Q, *)$ is a commutative monoid.

(2) $(N Q, \star)$ is a commutative monoid.

Theorem 1. [17] For the algebra system $(N Q, *)($ or $(N Q, \star))$, for every element a $\in N Q$, there exists the neutral element neut $(a)$ and opposite element anti $(a)$, which means that the algebra system $(N Q, *)$ (or $(N Q, \star))$ is a NETG.

\section{The Algebra Structure of Neutrosophic Quadruple Numbers on General Field}

From the above section, we can see that the neutrosophic quadruple numbers are defined on number field $\mathbb{R}$ or $\mathbb{C}$. In this section, the notions of the neutrosophic quadruple numbers on a general field are introduced and the algebra structure of the neutrosophic quadruple numbers on general field is explored.

Let $(\mathbb{F},+, \cdot)$ be a field, and 0 and 1 are the unit elements for operator + and $\cdot$, respectively. For every $a \in \mathbb{F},-a$ is the inverse element of $a$ for operator + , and $a^{-1}$ is the inverse element of $a$ for operator $\cdot$. In the following, field $(\mathbb{F},+, \cdot)$ is denoted by $\mathbb{F}$ for short and $a \cdot b$ is denoted by $a b$.

Definition 8. Let $\mathbb{F}$ be a field; a neutrosophic quadruple number is a number of the form $(a, b T, c I, d F)$, where $T, I, F$ have their usual neutrosophic logic meanings, i.e., truth, indeterminacy and false, respectively, and $a, b, c, d \in \mathbb{F}$. The set NQF defined by

$$
N Q F=\{(a, b T, c I, d F) \mid a, b, c, d \in \mathbb{F}\} .
$$

is called a neutrosophic set of quadruple numbers on field $\mathbb{F}$.

Definition 9. Let $a=\left(a_{1}, a_{2} T, a_{3} I, a_{4} F\right), b=\left(b_{1}, b_{2} T, b_{3} I, b_{4} F\right) \in N Q F$, then the addition operator is defined as follows:

$$
a \oplus b=\left(a_{1}+b_{1},\left(a_{2}+b_{2}\right) T,\left(a_{3}+b_{3}\right) I,\left(a_{4}+b_{4}\right) F\right) .
$$

Definition 10. Let $a=\left(a_{1}, a_{2} T, a_{3} I, a_{4} F\right), b=\left(b_{1}, b_{2} T, b_{3} I, b_{4} F\right) \in N Q F$. Suppose, in an pessimistic way, the neutrosophic expert considers the prevalence order $T \prec I \prec F$. Then, the multiplication operation is defined as follows:

$$
\begin{aligned}
a * b= & \left(a_{1}, a_{2} T, a_{3} I, a_{4} F\right) *\left(b_{1}, b_{2} T, b_{3} I, b_{4} F\right) \\
= & \left(a_{1} b_{1},\left(a_{1} b_{2}+a_{2} b_{1}+a_{2} b_{2}\right) T,\left(a_{1} b_{3}+a_{2} b_{3}+a_{3} b_{1}+a_{3} b_{2}+a_{3} b_{3}\right) I,\right. \\
& \left.\left(a_{1} b_{4}+a_{2} b_{4}+a_{3} b_{4}+a_{4} b_{1}+a_{4} b_{2}+a_{4} b_{3}+a_{4} b_{4}\right) F\right) .
\end{aligned}
$$

Suppose in an optimistic way the neutrosophic expert considers the prevalence order $T \succ I \succ F$. Then,

$$
\begin{aligned}
a \star b= & \left(a_{1}, a_{2} T, a_{3} I, a_{4} F\right) \star\left(b_{1}, b_{2} T, b_{3} I, b_{4} F\right) \\
= & \left(a_{1} b_{1},\left(a_{1} b_{2}+a_{2} b_{1}+a_{2} b_{2}+a_{3} b_{2}+a_{4} b_{2}+a_{2} b_{3}+a_{2} b_{4}\right) T,\right. \\
& \left.\left(a_{1} b_{3}+a_{3} b_{1}+a_{3} b_{3}+a_{3} b_{4}+a_{4} b_{3}\right) I,\left(a_{1} b_{4}+a_{4} b_{1}+a_{4} b_{4}\right) F\right) .
\end{aligned}
$$

Theorem 2. $(N Q F, \oplus)$ is an abelian group.

Proof. It is obvious.

Theorem 3. For the algebra system $(N Q F, *)$ (or $(N Q F, \star))$, for every element a $\in N Q F$, there exists the neutral element neut $(a)$ and opposite element anti $(a)$, thus the algebra system $(N Q F, *)($ or $(N Q F, \star))$ is a NETG. 
The proof's method is similar to the proof when $\mathbb{F}=\mathbb{R}$ in [17]. The detailed proof is omitted. For algebra system $(N Q F, *)$, Table 1 gives all the subsets which have the same neutral element, and the corresponding neutral element and opposite elements. In the following, from two examples, we show that how to solve the the neutral element and opposite elements of each element for algebra system $(N Q F, *)$ on different fields.

Example 1. Let $\mathbb{F}=Z_{5}=\{[0],[1],[2],[3],[4]\}$, then $\left(Z_{5},+, \cdot\right)$ is a field, where + and $\cdot$ are the classical mod addition and multiplication, respectively. For algebra system $(N Q F, *)$, if $a=\left(a_{1}, a_{2} T, a_{3} I, a_{4} F\right)=$ $([2],[4] T,[3] I,[1] F)$, i.e., $a_{1} \neq[0], a_{1}+a_{2} \neq[0], a_{1}+a_{2}+a_{3} \neq[0], a_{1}+a_{2}+a_{3}+a_{4}=[0]$, then, from Table 1, we can get neut $(a)=([1],[0],[0],[4] F)$. Let anti $(a)=\left(c_{1}, c_{2} T, c_{3} I, c_{4} F\right)$, so $c_{1}=a_{1}^{-1}=[3], c_{2}=[3], c_{3}=[3], c_{4} \in Z_{5}$, thus anti $(a)=\left([3],[3] T,[3] I, c_{4} F\right)$, where $c_{4} \in Z_{5}$. Thus, we can easily get the neutral element and opposite elements of each neutrosophic quadruple number on general field. For more examples, see the following:

1. Let $b=([1],[2] T,[1] I,[3] F)$, then neut $(b)=([1],[0],[0],[0])$ and anti $(b)=([1],[1] T,[2] I,[4] F)$.

2. Let $c=([0],[0],[1] I,[4] F)$, then neut $(c)=([0],[0],[1],[4])$ and anti $(c)=\left(c_{1}, c_{2} T, c_{3} I, c_{4} F\right)$, where $c_{1} \oplus c_{2} \oplus c_{3}=[1], c_{4} \in Z_{5}$.

3. Let $d=([0],[1] T,[1] I,[1] F)$, then neut $(d)=([0],[1] T,[0],[0])$ and anti $(d)=\left(c_{1}, c_{2} T,[2] I,[4] F\right)$, where $c_{1} \oplus c_{2}=[1]$.

Table 1. The corresponding neutral element and opposite elements for $(N Q F, *)$.

\begin{tabular}{|c|c|c|}
\hline The Subset of NQF & $\begin{array}{l}\text { Neutral } \\
\text { Element }\end{array}$ & Opposite Elements $\left(c_{1}, c_{2} T, c_{3} I, c_{4} F\right)$ \\
\hline$\{(0,0,0,0)\}$ & $(0,0,0,0)$ & $c_{i} \in \mathbb{F}$ \\
\hline$\left\{\left(0,0,0, a_{4} F\right) \mid a_{4} \neq 0\right\}$ & $(0,0,0, F)$ & $c_{1}+c_{2}+c_{3}+c_{4}=a_{4}^{-1}$ \\
\hline$\left\{\left(0,0, a_{3} I,-a_{3} F\right) \mid a_{3} \neq 0\right\}$ & $(0,0, I,-F)$ & $c_{1}+c_{2}+c_{3}=a_{3}^{-1}, c_{4} \in \mathbb{F}$ \\
\hline$\left\{\left(0,0, a_{3} I, a_{4} F\right) \mid a_{3} \neq 0, a_{3}+a_{4} \neq 0\right\}$ & $(0,0, I, 0)$ & $c_{1}+c_{2}+c_{3}=a_{3}^{-1}, c_{4}=-\left(a_{4} a_{3}^{-1}\left(a_{3}+a_{4}\right)^{-1}\right)$ \\
\hline$\left\{\left(0, a_{2} T,-a_{2} I, 0\right) \mid a_{2} \neq 0\right\}$ & $(0, T,-I, 0)\}$ & $c_{1}+c_{2}=a_{2}^{-1}, c_{3}, c_{4} \in \mathbb{F}$ \\
\hline$\left\{\left(0, a_{2} T,-a_{2} I, a_{4} F\right) \mid a_{2} \neq 0, a_{4} \neq 0\right\}$ & $(0, T,-I, F)$ & $c_{1}+c_{2}=a_{2}^{-1}, c_{3}+c_{4}=a_{4}^{-1}+\left(-a_{2}^{-1}\right)$ \\
\hline $\begin{array}{l}\left\{\left(0, a_{2} T, a_{3} I, a_{4} F\right) \mid a_{2} \neq 0, a_{2}+a_{3} \neq\right. \\
\left.0, a_{2}+a_{3}+a_{4}=0\right\}\end{array}$ & $(0, T, 0,-F)$ & $\begin{array}{l}c_{1}+c_{2}=a_{2}^{-1}, c_{3}=-\left(a_{3} a_{2}^{-1}\left(a_{2}+a_{3}\right)^{-1}\right) \\
c_{4} \in \mathbb{F}\end{array}$ \\
\hline $\begin{array}{l}\left\{\left(0, a_{2} T, a_{3} I, a_{4} F\right) \mid a_{2} \neq 0, a_{2}+a_{3} \neq\right. \\
\left.0, a_{2}+a_{3}+a_{4} \neq 0\right\}\end{array}$ & $(0, T, 0,0)$ & $\begin{array}{l}c_{1}+c_{2}=a_{2}^{-1}, c_{3}=-\left(a_{3} a_{2}^{-1}\left(a_{2}+a_{3}\right)^{-1}\right) \\
c_{4}=-\left(a_{4}\left(a_{2}+a_{3}\right)^{-1}\left(a_{2}+a_{3}+a_{4}\right)^{-1}\right)\end{array}$ \\
\hline$\left\{\left(a_{1},-a_{1} T, 0,0\right) \mid a_{1} \neq 0\right\}$ & $(1,-T, 0,0)\}$ & $c_{1}=a_{1}^{-1}, c_{2}, c_{3}, c_{4} \in \mathbb{F}$ \\
\hline$\left\{\left(a_{1},-a_{1} T, 0, a_{4} F\right) \mid a_{1} \neq 0, a_{4} \neq 0\right\}$ & $(1,-T, 0, F)$ & $c_{1}=a_{1}^{-1}, c_{2}+c_{3}+c_{4}=a_{4}^{-1}+\left(-a_{1}^{-1}\right)$ \\
\hline$\left\{\left(a_{1},-a_{1} T, a_{3} I,-a_{3} F\right) \mid a_{1} \neq 0, a_{3} \neq 0\right\}$ & $(1,-T, I,-F)$ & $c_{1}=a_{1}^{-1}, c_{2}+c_{3}=a_{3}^{-1}-a_{1}^{-1}, c_{4} \in \mathbb{F}$ \\
\hline $\begin{array}{l}\left\{\left(a_{1},-a_{1} T, a_{3} I, a_{4} F\right) \mid a_{1} \neq 0, a_{3} \neq\right. \\
\left.0, a_{3}+a_{4} \neq 0\right\}\end{array}$ & $(1,-T, I, 0)$ & $\begin{array}{l}c_{1}=a_{1}^{-1}, c_{2}+c_{3}=a_{3}^{-1}+\left(-a_{1}^{-1}\right) \\
c_{4}=-\left(a_{4} a_{3}^{-1}\left(a_{3}+a_{4}\right)^{-1}\right)\end{array}$ \\
\hline $\begin{array}{l}\left\{\left(a_{1}, a_{2} T, a_{3} I, 0\right) \mid a_{1} \neq 0, a_{1}+a_{2} \neq\right. \\
\left.0, a_{1}+a_{2}+a_{3}=0\right\}\end{array}$ & $(1,0,-I, 0)$ & $\begin{array}{l}c_{1}=a_{1}^{-1}, c_{2}=-\left(a_{2} a_{1}^{-1}\left(a_{1}+a_{2}\right)^{-1}\right), \\
c_{3}, c_{4} \in \mathbb{F}\end{array}$ \\
\hline $\begin{array}{l}\left\{\left(a_{1}, a_{2} T, a_{3} I, a_{4} F\right) \mid a_{1} \neq 0, a_{1}+a_{2} \neq\right. \\
\left.0, a_{1}+a_{2}+a_{3}=0, a_{4} \neq 0\right\}\end{array}$ & $(1,0,-I, F)$ & $\begin{array}{l}c_{1}=a_{1}^{-1}, c_{2}=-\left(a_{2} a_{1}^{-1}\left(a_{1}+a_{2}\right)^{-1}\right), \\
c_{3}+c_{4}=a_{4}^{-1}+\left(-\left(a_{1}+a_{2}\right)^{-1}\right)\end{array}$ \\
\hline $\begin{array}{l}\left\{\left(a_{1}, a_{2} T, a_{3} I, a_{4} F\right) \mid a_{1} \neq 0, a_{1}+a_{2} \neq\right. \\
0, a_{1}+a_{2}+a_{3} \neq 0, a_{1}+a_{2}+a_{3}+a_{4}= \\
0\}\end{array}$ & $(1,0,0,-F)$ & $\begin{array}{l}c_{1}=a_{1}^{-1}, c_{2}=-\left(a_{2} a_{1}^{-1}\left(a_{1}+a_{2}\right)^{-1}\right), \\
c_{3}=-\left(a_{3}\left(a_{1}+a_{2}\right)^{-1}\left(a_{1}+a_{2}+a_{3}\right)^{-1}\right), \\
c_{4} \in \mathbb{F}\end{array}$ \\
\hline $\begin{array}{l}\left\{\left(a_{1}, a_{2} T, a_{3} I, a_{4} F\right) \mid a_{1} \neq 0, a_{1}+a_{2} \neq\right. \\
0, a_{1}+a_{2}+a_{3} \neq 0, a_{1}+a_{2}+a_{3}+a_{4} \neq \\
0\}\end{array}$ & $(1,0,0,0)$ & $\begin{array}{l}c_{1}=a_{1}^{-1}, c_{2}=-\left(a_{2} a_{1}^{-1}\left(a_{1}+a_{2}\right)^{-1}\right) \\
c_{3}=-\left(a_{3}\left(a_{1}+a_{2}\right)^{-1}\left(a_{1}+a_{2}+a_{3}\right)^{-1}\right) \\
c_{4}=-\left(a_{4}\left(a_{1}+a_{2}+a_{3}\right)^{-1}\left(a_{1}+a_{2}+a_{3}+a_{4}\right)^{-1}\right)\end{array}$ \\
\hline
\end{tabular}


Example 2. Let $\mathbb{F}_{4}=\{0,1, x, y\}$, the operators + and $\cdot$ on $\mathbb{F}_{4}$ is defined by Table 2 .

Table 2. The operators + and $\cdot$ on $\mathbb{F}_{4}$.

\begin{tabular}{llllllllll}
\hline+ & 0 & 1 & $x$ & $y$ & $\cdot$ & 0 & 1 & $x$ & $y$ \\
0 & 0 & 1 & $x$ & $y$ & 0 & 0 & 0 & 0 & 0 \\
1 & 1 & 0 & $y$ & $x$ & 1 & 0 & 1 & $x$ & $y$ \\
$x$ & $x$ & $y$ & 0 & 1 & $x$ & 0 & $x$ & $y$ & 1 \\
$y$ & $y$ & $x$ & 1 & 0 & $y$ & 0 & $y$ & 1 & $x$ \\
\hline
\end{tabular}

Then, $\left(\mathbb{F}_{4},+, \cdot\right)$ is a field. Set $N Q F=\left\{(a, b T, c I, d F) \mid a, b, c, d \in \mathbb{F}_{4}\right\}$. We have:

1. Let $a=(0,0, x I, x F)$, then neut $(a)=(0,0, I, F)$ and anti $(a)=\left(c_{1}, c_{2} T, c_{3} I, c_{4} F\right)$, where $c_{1}+c_{2}+$ $c_{3}=y, c_{4} \in \mathbb{F}_{4}$.

2. Let $b=(0, x T, x I, y F)$, then neut $(b)=(0, T, I, F)$ and anti $(b)=\left(c_{1}, c_{2} T, c_{3} I, c_{4} F\right)$, where $c_{1}+c_{2}=$ $y, c_{3}+c_{4}=1$.

3. Let $c=(x, x T, 0,0)$, then neut $(c)=(1, T, 0,0)$ and anti $(c)=\left(y, c_{2} T, c_{3} I, c_{4} F\right)$, where $c_{2}, c_{3}, c_{4} \in \mathbb{F}_{4}$.

In the same way, for algebra system $(N Q F, \star)$, Table 3 gives all the subsets which have the same neutral element, and the corresponding neutral element and opposite elements.

Table 3. The corresponding neutral element and opposite elements for $(N Q F, \star)$.

\begin{tabular}{|c|c|c|}
\hline The Subset of NQF & $\begin{array}{l}\text { Neutral } \\
\text { Element }\end{array}$ & Opposite Elements $\left(c_{1}, c_{2} T, c_{3} I, c_{4} F\right)$ \\
\hline$\{(0,0,0,0)\}$ & $(0,0,0,0)$ & $c_{i} \in \mathbb{F}$ \\
\hline$\left\{\left(0, a_{2} T, 0,0\right) \mid a_{2} \neq 0\right\}$ & $(0, T, 0,0)$ & $c_{1}+c_{2}+c_{3}+c_{4}=a_{2}^{-1}$ \\
\hline$\left\{\left(0,-a_{3} T, a_{3} I, 0\right) \mid a_{3} \neq 0\right\}$ & $(0,-T, I, 0)$ & $c_{1}+c_{3}+c_{4}=a_{3}^{-1}, c_{2} \in \mathbb{F}$ \\
\hline$\left\{\left(0, a_{2} T, a_{3} I, a_{4} F\right) \mid a_{3} \neq 0, a_{2}+a_{3} \neq 0\right\}$ & $(0,0, I, 0)$ & $\begin{array}{l}c_{1}+c_{3}+c_{4}=a_{3}^{-1} \\
c_{2}=-\left(a_{2} a_{3}^{-1}\left(a_{2}+a_{3}\right)^{-1}\right)\end{array}$ \\
\hline$\left\{\left(0,0,-a_{4} I, a_{4} F\right) \mid a_{4} \neq 0\right\}$ & $(0,0,-I, F)\}$ & $c_{1}+c_{4}=a_{4}^{-1}, c_{2}, c_{3} \in \mathbb{F}$ \\
\hline$\left\{\left(0, a_{2} T,-a_{4} I, a_{4} F\right) \mid a_{2} \neq 0, a_{4} \neq 0\right\}$ & $(0, T,-I, F)$ & $c_{1}+c_{4}=a_{4}^{-1}, c_{2}+c_{3}=a_{2}^{-1}+\left(-a_{4}^{-1}\right)$ \\
\hline $\begin{array}{l}\left\{\left(0, a_{2} T, a_{3} I, a_{4} F\right) \mid a_{4} \neq 0, a_{3}+a_{4} \neq\right. \\
\left.0, a_{2}+a_{3}+a_{4}=0\right\}\end{array}$ & $(0,-T, 0, F)$ & $\begin{array}{l}c_{1}+c_{4}=a_{4}^{-1}, c_{3}=-\left(a_{3} a_{4}^{-1}\left(a_{3}+a_{4}\right)^{-1}\right) \\
c_{2} \in \mathbb{F}\end{array}$ \\
\hline $\begin{array}{l}\left\{\left(0, a_{2} T, a_{3} I, a_{4} F\right) \mid a_{4} \neq 0, a_{3}+a_{4} \neq\right. \\
\left.0, a_{2}+a_{3}+a_{4} \neq 0\right\}\end{array}$ & $(0,0,0, F)$ & $\begin{array}{l}c_{1}+c_{4}=a_{4}^{-1}, c_{3}=-\left(a_{3} a_{4}^{-1}\left(a_{3}+a_{4}\right)^{-1}\right) \\
c_{2}=-\left(a_{2}\left(a_{3}+a_{4}\right)^{-1}\left(a_{2}+a_{3}+a_{4}\right)^{-1}\right)\end{array}$ \\
\hline$\left\{\left(a_{1}, 0,0,-a_{1} F\right) \mid a_{1} \neq 0\right\}$ & $(1,0,0,-F)\}$ & $c_{1}=a_{1}^{-1}, c_{2}, c_{3}, c_{4} \in \mathbb{F}$ \\
\hline$\left\{\left(a_{1}, a_{2} T, 0,-a_{1} F\right) \mid a_{1} \neq 0, a_{2} \neq 0\right\}$ & $(1, T, 0,-F)$ & $c_{1}=a_{1}^{-1}, c_{2}+c_{3}+c_{4}=a_{2}^{-1}+\left(-a_{1}^{-1}\right)$ \\
\hline$\left\{\left(a_{1},-a_{3} T, a_{3} I,-a_{1} F\right) \mid a_{1} \neq 0, a_{3} \neq 0\right\}$ & $(1,-T, I,-F)$ & $c_{1}=a_{1}^{-1}, c_{3}+c_{4}=a_{3}^{-1}+\left(-a_{1}^{-1}\right), c_{4} \in \mathbb{F}$ \\
\hline $\begin{array}{l}\left\{\left(a_{1}, a_{2} T, a_{3} I,-a_{1} F\right) \mid a_{1} \neq 0, a_{3} \quad \neq\right. \\
\left.0, a_{2}+a_{3} \neq 0\right\}\end{array}$ & $(1,0, I,-F)$ & $\begin{array}{l}c_{1}=a_{1}^{-1}, c_{3}+c_{4}=a_{3}^{-1}+\left(-a_{1}^{-1}\right) \\
c_{2}=-\left(a_{2} a_{3}^{-1}\left(a_{2}+a_{3}\right)^{-1}\right)\end{array}$ \\
\hline $\begin{array}{l}\left\{\left(a_{1}, 0, a_{3} I, a_{4} F\right) \mid a_{1} \neq 0, a_{1}+a_{4} \neq\right. \\
\left.0, a_{1}+a_{3}+a_{4}=0\right\}\end{array}$ & $(1,0,-I, 0)$ & $\begin{array}{l}c_{1}=a_{1}^{-1}, c_{4}=-\left(a_{4} a_{1}^{-1}\left(a_{1}+a_{4}\right)^{-1}\right) \\
c_{2}, c_{3} \in \mathbb{F}\end{array}$ \\
\hline $\begin{array}{l}\left\{\left(a_{1}, a_{2} T, a_{3} I, a_{4} F\right) \mid a_{1} \neq 0, a_{1}+a_{4} \neq\right. \\
\left.0, a_{1}+a_{3}+a_{4}=0, a_{2} \neq 0\right\}\end{array}$ & $(1, T,-I, 0)$ & $\begin{array}{l}c_{1}=a_{1}^{-1}, c_{4}=-\left(a_{4} a_{1}^{-1}\left(a_{1}+a_{4}\right)^{-1}\right) \\
c_{2}+c_{3}=a_{2}^{-1}+\left(-\left(a_{1}+a_{4}\right)^{-1}\right)\end{array}$ \\
\hline $\begin{array}{l}\left\{\left(a_{1}, a_{2} T, a_{3} I, a_{4} F\right) \mid a_{1} \neq 0, a_{1}+a_{4} \neq\right. \\
0, a_{1}+a_{3}+a_{4} \neq 0, a_{1}+a_{2}+a_{3}+a_{4}= \\
0\}\end{array}$ & $(1,-T, 0,0)$ & $\begin{array}{l}c_{1}=a_{1}^{-1}, c_{4}=-\left(a_{4} a_{1}^{-1}\left(a_{1}+a_{4}\right)^{-1}\right), \\
c_{3}=-\left(a_{3}\left(a_{1}+a_{4}\right)^{-1}\left(a_{1}+a_{3}+a_{4}\right)^{-1}\right), \\
c_{2} \in \mathbb{F}\end{array}$ \\
\hline $\begin{array}{l}\left\{\left(a_{1}, a_{2} T, a_{3} I, a_{4} F\right) \mid a_{1} \neq 0, a_{1}+a_{4} \neq\right. \\
0, a_{1}+a_{3}+a_{4} \neq 0, a_{1}+a_{2}+a_{3}+a_{4} \neq \\
0\}\end{array}$ & $(1,0,0,0)$ & $\begin{array}{l}c_{1}=a_{1}^{-1}, c_{4}=-\left(a_{4} a_{1}^{-1}\left(a_{1}+a_{4}\right)^{-1}\right) \\
c_{3}=-\left(a_{3}\left(a_{1}+a_{4}\right)^{-1}\left(a_{1}+a_{3}+a_{4}\right)^{-1}\right) \\
c_{2}=-\left(a_{2}\left(a_{1}+a_{3}+a_{4}\right)^{-1}\left(a_{1}+a_{2}+a_{3}+a_{4}\right)^{-1}\right)\end{array}$ \\
\hline
\end{tabular}




\section{The Algebra Structure of Refined Neutrosophic Numbers on General Field}

In the above section, we reveal that the algebra structure of the neutrosophic quadruple numbers on general field. In this section, we explore the the algebra structure of the refined neutrosophic numbers (refined neutrosophic quadruple numbers) on general field.

Definition 11. Let $\mathbb{F}$ be a field; a refined n-ary neutrosophic number is a number of the form $\left(a_{0}, a_{1} I_{1}, a_{2} I_{2}, \cdots, a_{n} I_{n}\right)$, where $I_{1}, I_{2}, \cdots$, and $I_{n}$ are different types of indeterminacies, and $a_{0}, a_{1}, a_{2}, \cdots, a_{n} \in \mathbb{F}$. The set $R N F_{n}$ defined by

$$
R N F_{n}=\left\{\left(a_{0}, a_{1} I_{1}, a_{2} I_{2}, \cdots, a_{n} I_{n}\right) \mid a_{0}, a_{1}, a_{2}, \cdots, a_{n} \in \mathbb{F}\right\} .
$$

is called a refined neutrosophic set on field $\mathbb{F}$.

Definition 12. Let $x=\left(a_{0}, a_{1} I_{1}, a_{2} I_{2}, \cdots, a_{n} I_{n}\right), y=\left(b_{0}, b_{1} I_{1}, b_{2} I_{2}, \cdots, b_{n} I_{n}\right) \in R N F_{n}$, then the addition operator on $R N F_{n}$ is defined as follows:

$$
x \oplus y=\left(a_{0}+b_{0},\left(a_{1}+b_{1}\right) I_{1},\left(a_{2}+b_{2}\right) I_{2}, \cdots,\left(a_{n}+b_{n}\right) I_{n}\right) .
$$

Definition 13. Let $x=\left(a_{0}, a_{1} I_{1}, a_{2} I_{2}, \cdots, a_{n} I_{n}\right), y=\left(b_{0}, b_{1} I_{1}, b_{2} I_{2}, \cdots, b_{n} I_{n}\right) \in R N F_{n}$, the neutrosophic expert considers the prevalence order $I_{1} \prec I_{2} \prec \cdots \prec I_{n}$. Then, the multiplication operation is defined as follows:

$$
\begin{aligned}
x * y= & \left(a_{0}, a_{1} I_{1}, a_{2} I_{2}, \cdots, a_{n} I_{n}\right) *\left(b_{0}, b_{1} I_{1}, b_{2} I_{2}, \cdots, b_{n} I_{n}\right) \\
= & \left(a_{0} b_{0},\left(a_{0} b_{1}+a_{1} b_{1}+a_{1} b_{2}\right) I_{1},\left(a_{0} b_{2}+a_{1} b_{2}+a_{2} b_{0}+a_{2} b_{1}+a_{2} b_{2}\right) I_{2},\right. \\
& \left.\cdots,\left(a_{0} b_{n}+a_{1} b_{n}+a_{2} b_{n}+\cdots+a_{n-1} b_{n}+a_{n} b_{0}+a_{n} b_{1}+\cdots+a_{n} b_{n}\right) I_{n}\right) .
\end{aligned}
$$

The neutrosophic expert considers the prevalence order $I_{1} \succ I_{2} \succ \cdots \succ I_{n}$. Then,

$$
\begin{aligned}
x \star y= & \left(a_{0}, a_{1} I_{1}, a_{2} I_{2}, \cdots, a_{n} I_{n}\right) \star\left(b_{0}, b_{1} I_{1}, b_{2} I_{2}, \cdots, b_{n} I_{n}\right) \\
= & \left(a_{0} b_{0},\left(a_{0} b_{1}+a_{1} b_{1}+\cdots+a_{n} b_{1}+a_{1} b_{0}+a_{1} b_{2}+a_{1} b_{3}+\cdots+a_{1} b_{n}\right) I_{1}, \cdots,\right. \\
& \left.\left(a_{0} b_{n-1}+a_{n-1} b_{0}+a_{n-1} b_{n-1}+a_{n-1} b_{n}+a_{n} b_{n-1}\right) I_{n-1},\left(a_{0} b_{n}+a_{n} b_{0}+a_{n} b_{n}\right) I_{n}\right) .
\end{aligned}
$$

Theorem 4. $\left(R N F_{n}, \oplus\right)$ is an abelian group.

Proof. The proof is obvious.

Theorem 5. For the algebra system $\left(R N F_{n}, *\right)$ (or $\left.\left(R N F_{n}, \star\right)\right)$, for every element $a \in R N F_{n}$, there exists the neutral element neut $(a)$ and opposite element anti $(a)$, thus the algebra system $\left(R N F_{n}, *\right)$ (or $\left.\left(R N F_{n}, \star\right)\right)$ is a NETG.

Proof. We use applied mathematical induction for $n$ and only discuss the algebra system $\left(R N F_{n}, *\right)$. The algebra system $\left(R N F_{n}, \star\right)$ has a similar proof.

If $n=2$, for refined 2-ary neutrosophic set, which is same as neutrosophic binary numbers set in [17], from Theorem 7 in [17], we can see that for every element $a \in R N F_{2}$, there exists the neutral element neut $(a)$ and opposite element anti $(a)$, thus the algebra system $\left(R N F_{2}, *\right)$ is a NETG.

Assume that the refined $n$-ary neutrosophic set $R N F_{n}$ is a NETG. That is, for every element $a \in R N F_{n}$, there exists the neutral element neut(a) and opposite element anti(a). In the following, we prove that for the refined $(n+1)$-ary neutrosophic set, which is a NETG.

For each $a=\left(a_{0}, a_{1} I_{1}, a_{2} I_{2}, \cdots, a_{n+1} I_{n+1}\right) \in R N F_{n+1}$, let $a^{\prime}=\left(a_{0}, a_{1} I_{1}, a_{2} I_{2}, \cdots, a_{n} I_{n}\right)$, being $a^{\prime} \in R N F_{n}$, then, from the above assumption condition, neut $\left(a^{\prime}\right)$ and anti $\left(a^{\prime}\right)$ exist and let neut $\left(a^{\prime}\right)=\left(b_{0}, b_{1} I_{1}, b_{2} I_{2}, \cdots, b_{n} I_{n}\right)$, anti $\left(a^{\prime}\right)=\left(c_{0}, c_{1} I_{1}, c_{2} I_{2}, \cdots, c_{n} I_{n}\right)$. We prove for $a=\left(a_{0}, a_{1} I_{1}, a_{2} I_{2}, \cdots, a_{n+1} I_{n+1}\right)$, neut $(a)$ and anti $(a)$ exist. We discuss from the different cases of $a_{n+1}$. 
Case A: If $a_{n+1}=0$, Being $\left(a_{0}, a_{1} I_{1}, a_{2} I_{2}, \cdots, a_{n} I_{n}, 0\right) *\left(b_{0}, b_{1} I_{1}, b_{2} I_{2}, \cdots, b_{n} I_{n}, b_{n+1} I_{n+1}\right)=$ $\left(a_{0}, a_{1} I_{1}, a_{2} I_{2}, \cdots, a_{n} I_{n}, 0\right)$, that is $\left(a_{0}+a_{1}+\cdots+a_{n}\right) b_{n+1}=0$, thus we discuss from $a_{0}+a_{1}+\cdots+$ $a_{n}=0$ or $a_{0}+a_{1}+\cdots+a_{n} \neq 0$.

Case A1: If $a_{n+1}=0, a_{0}+a_{1}+\cdots+a_{n}=0$, so $\left(a_{0}, a_{1} I_{1}, a_{2} I_{2}, \cdots, a_{n} I_{n}, 0\right) *$ $\left(c_{0}, c_{1} I_{1}, c_{2} I_{2}, \cdots, c_{n} I_{n}, c_{n+1} I_{n+1}\right)=\left(b_{0}, b_{1} I_{1}, b_{2} I_{2}, \cdots, b_{n} I_{n}, b_{n+1} I_{n+1}\right)$, that is $b_{n+1}=0$ and $c_{n+1}$ can be chosen arbitrarily in $\mathbb{F}$.

Case A2: If $a_{n+1}=0, a_{0}+a_{1}+\cdots+a_{n} \neq 0$, so from $\left(a_{0}+a_{1}+\cdots+a_{n}\right) b_{n+1}=0$, we have $b_{n+1}=0$, from $\left(a_{0}, a_{1} I_{1}, a_{2} I_{2}, \cdots, a_{n} I_{n}, 0\right) *\left(c_{0}, c_{1} I_{1}, c_{2} I_{2}, \cdots, c_{n} I_{n}, c_{n+1} I_{n+1}\right)=\left(b_{0}, b_{1} I_{1}, b_{2} I_{2}, \cdots, b_{n} I_{n}, 0\right)$, thus $c_{n+1}=0$.

Case B: If $a_{n+1} \neq 0$, being $\left(a_{0}, a_{1} I_{1}, a_{2} I_{2}, \cdots, a_{n} I_{n}, a_{n+1} I_{n+1}\right) *\left(b_{0}, b_{1} I_{1}, b_{2} I_{2}, \cdots, b_{n} I_{n}, b_{n+1} I_{n+1}\right)=$ $\left(a_{0}, a_{1} I_{1}, a_{2} I_{2}, \cdots, a_{n} I_{n}, a_{n+1} I_{n+1}\right)$, that is $\left(a_{0}+a_{1}+\cdots+a_{n+1}\right) b_{n+1}+a_{n+1}\left(b_{0}+b_{1}+\cdots+b_{n}\right)=a_{n+1}$, that is $\left(a_{0}+a_{1}+\cdots+a_{n+1}\right) b_{n+1}=a_{n+1}\left(1-b_{0}-b_{1}-\cdots-b_{n}\right)$, we discuss from $a_{0}+a_{1}+\cdots+a_{n+1}=0$ or $a_{0}+a_{1}+\cdots+a_{n+1} \neq 0$.

Case B1: If $a_{n+1} \neq 0, a_{0}+a_{1}+\cdots+a_{n+1}=0$, we have $b_{0}+b_{1}+\cdots+$ $b_{n}=1$, so from $\left(a_{0}, a_{1} I_{1}, a_{2} I_{2}, \cdots, a_{n} I_{n}, a_{n+1} I_{n+1}\right) *\left(c_{0}, c_{1} I_{1}, c_{2} I_{2}, \cdots, c_{n} I_{n}, c_{n+1} I_{n+1}\right)=$ $\left(b_{0}, b_{1} I_{1}, b_{2} I_{2}, \cdots, b_{n} I_{n}, b_{n+1} I_{n+1}\right)$, that is $b_{n+1}=\left(c_{0}+c_{1}+\cdots+c_{n}\right) a_{n+1}=b_{n+1}$ and $c_{n+1}$ can be chosen arbitrarily in $\mathbb{F}$.

Case B2: If $a_{n+1} \neq 0, a_{0}+a_{1}+\cdots+a_{n+1} \neq 0$, we have $b_{n+1}=a_{n+1}\left(1-b_{0}-b_{1}-\cdots-b_{n}\right)\left(a_{0}+\right.$ $\left.a_{1}+a_{2} \cdots a_{n}\right)^{-1}$, so from $\left(a_{0}, a_{1} I_{1}, a_{2} I_{2}, \cdots, a_{n} I_{n}, a_{n+1} I_{n+1}\right) *\left(c_{0}, c_{1} I_{1}, c_{2} I_{2}, \cdots, c_{n} I_{n}, c_{n+1} I_{n+1}\right)=$ $\left(b_{0}, b_{1} I_{1}, b_{2} I_{2}, \cdots, b_{n} I_{n}, b_{n+1} I_{n+1}\right)$, that is and $c_{n+1}=\left(b_{n+1}-a_{n+1}\left(c_{0}+c_{1}+\cdots+c_{n}\right)\right)\left(a_{0}+a_{1}+\cdots+\right.$ $\left.a_{n+1}\right)^{-1}$.

From the above analysis, we can see that, for each $a \in R N F_{n}$, which has the neutral element neut (a) and opposite element anti(a), from the mathematical induction method, we can obtain that the algebra system $(R N F, *)$ is a NETG.

For algebra system $\left(R N F_{n+1}, *\right)$, if $a=\left(a_{0}, a_{1} I_{1}, a_{2} I_{2}, \cdots, a_{n+1} I_{n+1}\right)$, let $a^{\prime}=$ $\left(a_{0}, a_{1} I_{1}, a_{2} I_{2}, \cdots, a_{n} I_{n}\right) \in R N F_{n}$, if we have neut $\left(a^{\prime}\right)=\left(b_{0}, b_{1} I_{1}, b_{2} I_{2}, \cdots, b_{n} I_{n}\right)$, anti $\left(a^{\prime}\right)=$ $\left(c_{0}, c_{1} I_{1}, c_{2} I_{2}, \cdots, c_{n} I_{n}\right)$. Then, the corresponding neutral element and opposite elements of $a$ are given in Table 4 according to the different cases of $a_{n+1}$.

Table 4. The corresponding neutral element and opposite elements for $\left(R N F_{n+1}, *\right)$

\begin{tabular}{lll}
\hline $\begin{array}{l}\text { The Subset } \\
\left\{\left(\boldsymbol{a}_{\mathbf{0}}, \boldsymbol{a}_{\mathbf{1}} \boldsymbol{I}_{\mathbf{1}}, \cdots, \boldsymbol{a}_{\boldsymbol{n}+\mathbf{1}} \boldsymbol{I}_{\boldsymbol{n}+\mathbf{1}}\right)\right\}\end{array}$ & $\begin{array}{l}\text { Neutral Element } \\
\left(\boldsymbol{b}_{\mathbf{0}}, \boldsymbol{b}_{\mathbf{1}} \boldsymbol{I}_{\mathbf{1}}, \cdots, \boldsymbol{b}_{\boldsymbol{n}} \boldsymbol{I}_{\boldsymbol{n}}, \boldsymbol{b}_{\boldsymbol{n}+\mathbf{1}} \boldsymbol{I}_{\boldsymbol{n}+\mathbf{1}}\right)\end{array}$ & $\begin{array}{l}\text { Opposite Elements } \\
\left(\boldsymbol{c}_{\mathbf{0}}, \boldsymbol{c}_{\mathbf{1}} \boldsymbol{I}_{\mathbf{1}}, \cdots, \boldsymbol{c}_{\boldsymbol{n}} \boldsymbol{I}_{\boldsymbol{n}}, \boldsymbol{c}_{\boldsymbol{n}+\mathbf{1}} \boldsymbol{I}_{\boldsymbol{n}+\mathbf{1}}\right)\end{array}$ \\
\hline $\begin{array}{l}a_{n+1}=0, \\
a_{0}+a_{1}+\cdots+a_{n}=0\end{array}$ & $b_{n+1}=0$ & $c_{n+1} \in \mathbb{F}$ \\
\hline $\begin{array}{l}a_{n+1}=0, \\
a_{0}+a_{1}+\cdots+a_{n} \neq 0\end{array}$ & $b_{n+1}=0$ & \\
\hline $\begin{array}{l}a_{n+1} \neq 0, \\
a_{0}+a_{1}+\cdots+a_{n}+a_{n+1}=0\end{array}$ & $c_{n+1} \in \mathbb{F}$ \\
\hline $\begin{array}{l}a_{n+1} \neq 0, \\
a_{0}+a_{1}+\cdots+a_{n}+a_{n+1} \neq 0\end{array}$ & $\begin{array}{l}b_{n+1}=\left(c_{0}+c_{1}+\cdots+c_{n}\right) a_{n+1} \\
\left.b_{n}\right)\left(a_{0}+a_{n+1}\left(1-a_{2}+\cdots+b_{1}-\cdots-a_{n}\right)^{-1}\right)\end{array}$ & $\begin{array}{l}c_{n+1}=\left(b_{n+1}-a_{n+1}\left(c_{0}+c_{1}+\cdots+\right.\right. \\
\left.\left.c_{n}\right)\right)\left(a_{0}+a_{1}+\cdots+a_{n+1}\right)^{-1}\end{array}$ \\
\hline
\end{tabular}

For algebra system $\left(R N F_{n}, *\right)$, according to the results in Tables 3 and 4 in [17], we can easily obtain the neutral element and opposite elements when $n=1,2$ on general fields. In Table 1 , we can get the neutral element and opposite elements when $n=3$ on general field. Thus, from Theorem 5 , we can get the neutral element and opposite elements of each element in $R N F_{n}$ step-by-step. The solving method is given by Algorithm 1 and the following example is used to explain the algorithm. 


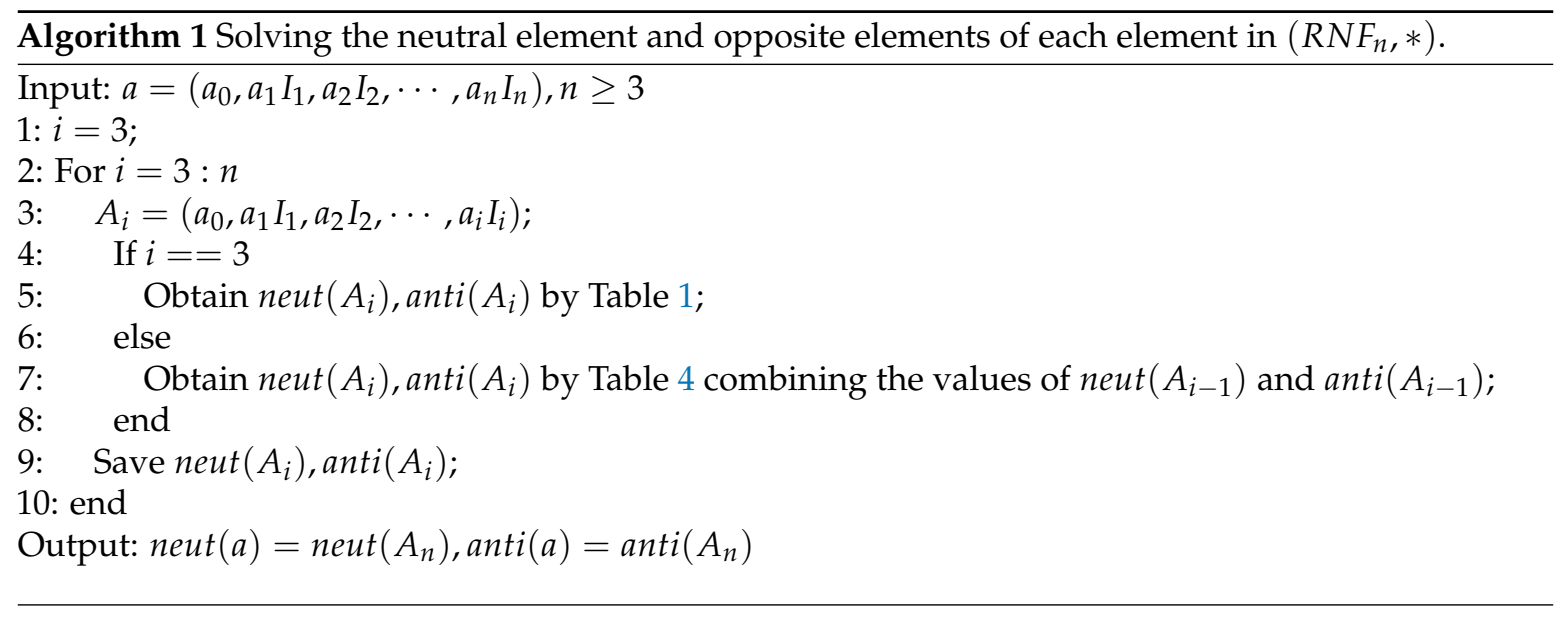

Example 3. For algebra system $\left(R N F_{2}, *\right)$, and set $\mathbb{F}=\mathbb{R}$. If $a=\left(a_{0}, a_{1} I_{1}\right)=\left(0,-I_{1}\right)$, from Table 3 in [17], we can get neut $(a)=\left(0, I_{1}\right)$ and anti $(a)=\left(c_{0}, c_{1} I_{1}\right)$, where $c_{0}+c_{1}=-1$.

In the following, we use two methods to solve the the neutral element and opposite elements of $b=$ $\left(a_{0}, a_{1} I_{1}, a-2 I_{2}\right)=\left(0,-I_{1}, I_{2}\right) \in R N F_{3}$ and we get the same results.

1. Algorithm 1: From Table 2, being $a_{3} \neq 0$ and $a_{0}+a_{1}+a_{2}=0$, thus $b_{2}=\left(c_{0}+c_{1}\right) \cdot 1=-1$, that is neut $(b)=\left(0, I_{1},-I_{2}\right)$ and anti $(a)=\left(c_{0}, c_{1} I_{1}, c_{2} I_{2}\right)$, where $c_{0}+c_{1}=-1$ and $c_{2}$ can be chosen arbitrarily in $\mathbb{R}$.

2. Rsults from Table 4 in [17]: Being $a_{2} \neq 0$ and $a_{0}+a_{1}+a_{2}=0$, thus neut $(b)=\left(0, I_{1},-I_{2}\right)$ and anti $(a)=\left(c_{0}, c_{1} I_{1}, c_{2} I_{2}\right)$, where $c_{0}+c_{1}=-1$ and $c_{2}$ can be chosen arbitrarily in $\mathbb{R}$.

Example 4. For algebra system $\left(R N F_{3}, *\right)$, and set $\mathbb{F}=\mathbb{R}$. If $a=\left(a_{0}, a_{1} I_{1}, a_{2} I_{2}\right)=\left(1,-I_{1}, I_{2}\right) \in R N F_{3}$, from Table 4 in [17], we can get neut $(a)=\left(1,-I_{1}, I_{2}\right)$ and anti $(a)=\left(1, c_{1} I_{1}, c_{2} I_{2}\right)$, where $c_{1}+c_{2}=0$.

In the same way, we use two methods to solve the the neutral element and opposite elements of $b=$ $\left(a_{0}, a_{1} I_{1}, a_{2} I_{2}, a_{3} I_{3}\right)=\left(1,-I_{1}, I_{2}, I_{3}\right) \in R N F_{4}$.

1. Algorithm 1: From Table 2, being $a_{3} \neq 0$ and $a_{0}+a_{1}+a_{2}+a_{3} \neq 0$, thus $b_{3}=0$, that is neut $(b)=$ $\left(0,-I_{1}, I_{2}, 0\right)$ and $c_{3}=\left(0-1 \cdot 1 \cdot \frac{1}{2}\right)=-\frac{1}{2}$, thus anti $(a)=\left(1, c_{1} I_{1}, c_{2} I_{2},-\frac{1}{2} I_{3}\right)$, where $c_{1}+c_{2}=0$.

2. Results from Table 1 in [17]: Being $a_{0} \neq 0, a_{2} \neq 0$ and $a_{2}+a_{3} \neq 0$, thus neut $(b)=\left(1,-I_{1}, I_{2}, 0\right)$ and $c_{0}=1, c_{1}+c_{2}=0, c_{3}=-\frac{1}{1 \cdot(1+1)}=-\frac{1}{2}$, thus anti $(a)=\left(1, c_{1} I_{1}, c_{2} I_{2},-\frac{1}{2} I_{3}\right)$, where $c_{1}+c_{2}=0$.

For algebra system $\left(R N F_{n+1}, \star\right)$, set $a=\left(a_{0}, a_{1} I_{1}, a_{2} I_{2}, \cdots, a_{n+1} I_{n+1}\right)$, being the order $I_{1} \succ I_{2} \succ \cdots \succ I_{n}$, thus we should obtain the neutral element and opposite elements of $a^{\prime}=\left(a_{0}, a_{2} I_{2}, \cdots, a_{n+1} I_{n+1}\right)$. Knowing that neut $\left(a^{\prime}\right)=\left(b_{0}, b_{2} I_{2}, b_{3} I_{3}, \cdots, b_{n} I_{n}\right)$, anti $\left(a^{\prime}\right)=$ $\left(c_{0}, c_{2} I_{2}, c_{3} I_{3}, \cdots, c_{n} I_{n}\right)$, then the corresponding neutral element and opposite elements of $a$ are given in Table 5 according to the different cases of $a_{1}$. 
Table 5. The corresponding neutral element and opposite elements for $(R N F, \star)$.

\begin{tabular}{lll}
\hline $\begin{array}{l}\text { The Subset } \\
\left\{\left(\boldsymbol{a}_{\mathbf{0}}, \boldsymbol{a}_{\mathbf{1}} \boldsymbol{I}_{\mathbf{1}}, \cdots, \boldsymbol{a}_{\boldsymbol{n}+\mathbf{1}} \boldsymbol{I}_{\boldsymbol{n}+\mathbf{1}}\right)\right\}\end{array}$ & $\begin{array}{l}\text { Neutral Element } \\
\left(\boldsymbol{b}_{\mathbf{0}}, \boldsymbol{b}_{\mathbf{1}} \boldsymbol{I}_{\mathbf{1}}, \cdots, \boldsymbol{b}_{\boldsymbol{n}} \boldsymbol{I}_{\boldsymbol{n}}, \boldsymbol{b}_{\boldsymbol{n}+\mathbf{1}} \boldsymbol{I}_{\boldsymbol{n}+\mathbf{1}}\right)\end{array}$ & $\begin{array}{l}\text { Opposite Elements } \\
\left(\boldsymbol{c}_{\mathbf{0}}, \boldsymbol{c}_{\mathbf{1}} \boldsymbol{I}_{\mathbf{1}}, \cdots, \boldsymbol{c}_{\boldsymbol{n}} \boldsymbol{I}_{\boldsymbol{n}}, \boldsymbol{c}_{\boldsymbol{n}+\mathbf{1}} \boldsymbol{I}_{\boldsymbol{n}+\mathbf{1}}\right)\end{array}$ \\
\hline $\begin{array}{l}a_{1}=0, \\
a_{0}+a_{2}+\cdots+a_{n+1}=0\end{array}$ & $b_{1}=0$ & $c_{1} \in \mathbb{F}$ \\
\hline $\begin{array}{l}a_{1}=0, \\
a_{0}+a_{2}+\cdots+a_{n+1} \neq 0\end{array}$ & $b_{1}=0$ & $c_{1}=0$ \\
\hline $\begin{array}{l}a_{1} \neq 0, \\
a_{0}+a_{1}+\cdots+a_{n}+a_{n+1}=0\end{array}$ & $b_{1}=\left(c_{0}+c_{2}+\cdots+c_{n+1}\right) a_{1}$ & $c_{1} \in \mathbb{F}$ \\
\hline $\begin{array}{l}a_{1} \neq 0, \\
a_{0}+a_{1}+\cdots+a_{n}+a_{n+1} \neq 0\end{array}$ & $\left.\left.b_{1}=b_{n+1}\right)\right)\left(a_{0}+a_{1}+\cdots+b_{0}+b_{2}+\cdots+1\right)$ & $\begin{array}{l}c_{1}=\left(b_{1}-a_{1}\left(c_{0}+c_{2}+\cdots+\right.\right. \\
\left.\left.c_{n+1}\right)\right)\left(a_{0}+a_{1}+\cdots+a_{n+1}\right)^{-1}\end{array}$ \\
\hline
\end{tabular}

Similarly, we also can get the neutral element and opposite elements of each element in $\left(R N F_{n}, \star\right)$ step-by-step. The solving method is given by Algorithm 2 and the following example is used to explain the algorithm.

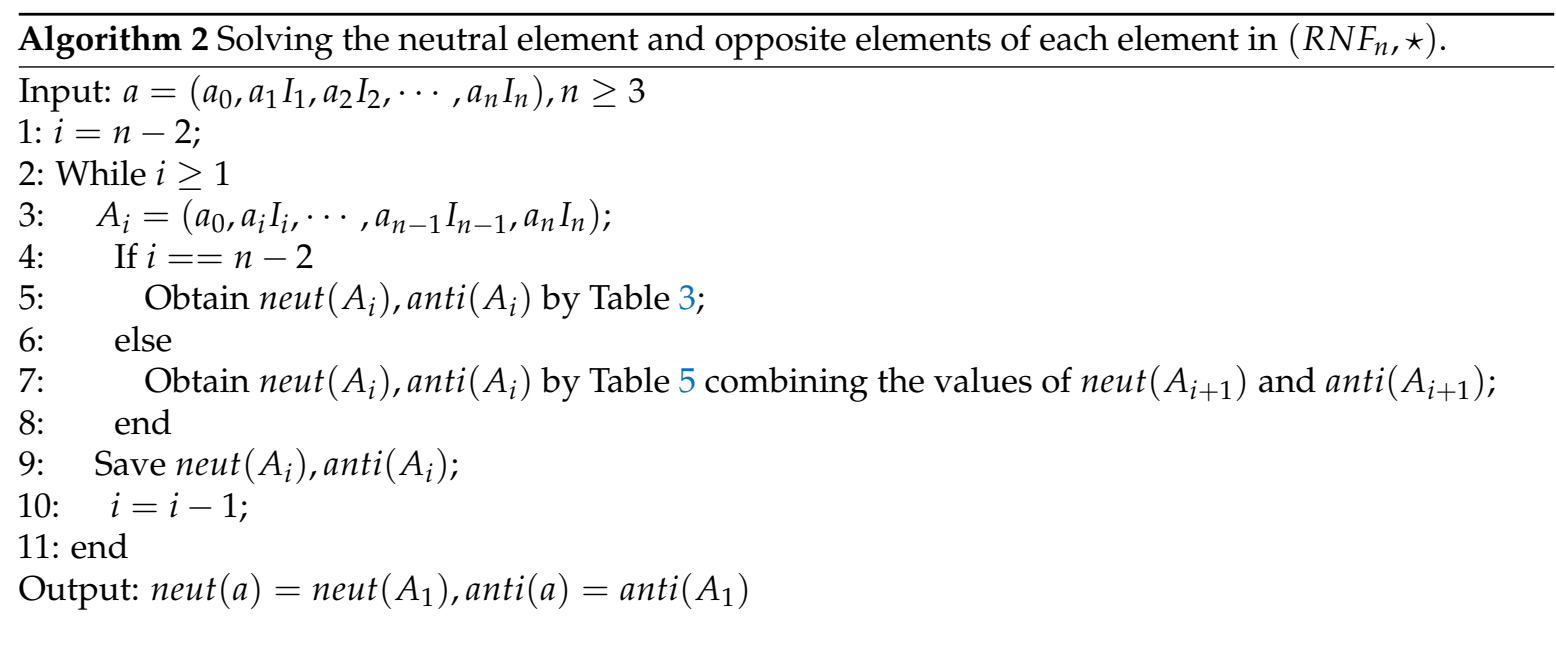

Example 5. For algebra system $\left(R N F_{6}, \star\right)$, and set $\mathbb{F}=\mathbb{R}, a=\left(0,0,-2 I_{2},-I_{3}, I_{4}, 0\right)$, solve the neutral element and opposite elements of $a$.

According Algorithm 2 for algebra system $\left(R N F_{6}, \star\right)$ : Firstly, we solve the neutral element and opposite elements of $a^{\prime}=\left(0,-I_{3}, I_{4}, 0\right)$ from Table 3 , and then solve the neutral element and opposite elements of $a^{\prime \prime}=\left(0,-2 I_{2},-I_{3}, I_{4}, 0\right)$ from Table 5 , lastly, we solve the neutral element and opposite elements of a from Table 5 .

1. From Table 3: neut $\left(a^{\prime}\right)=\left(0,-I_{3}, I_{4}, 0\right)$ and anti $\left(a^{\prime}\right)=\left(c_{0}, c_{3} I_{3}, c_{4} I_{4}, c_{5} I_{5}\right)$, where $c_{0}+c_{4}+c_{5}=1$, $c_{3} \in \mathbb{R}$.

2. From Table 5 and combining the results of the above step: Being $-2 \neq 0$ and $0+(-2)+(-1)+1+0 \neq$ 0 , thus neut $\left(a^{\prime \prime}\right)=\left(0, I_{2},-I_{3}, I_{4}, 0\right)$ and anti $\left(a^{\prime \prime}\right)=\left(c_{0}, c_{2} I_{2}, c_{3} I_{3}, c_{4} I_{4}, c_{5} I_{5}\right)$, where $c_{0}+c_{4}+c_{5}=1$, $c_{2}+c_{3}=-\frac{3}{2}$.

3. From Table 5 and combining the results of the above step: Being $0=0$ and $0+0+(-2)+(-1)+$ $1+0 \neq 0$, thus neut $(a)=\left(0,0, I_{2},-I_{3}, I_{4}, 0\right)$ and anti $(a)=\left(c_{0}, 0, c_{2} I_{2}, c_{3} I_{3}, c_{4} I_{4}, c_{5} I_{5}\right)$, where $c_{0}+c_{4}+c_{5}=1, c_{2}+c_{3}=-\frac{3}{2}$.

Similarly, we explore the the algebra structure of the refined neutrosophic quadruple numbers on general field in the following. 
Definition 14. Let $\mathbb{F}$ be a field; a refined neutrosophic quadruple number is a number of the form $\left(a, b_{1} T_{1}, b_{2} T_{2}, \cdots, b_{p} T_{p}, c_{1} I_{1}, c_{2} I_{2}, \cdots, c_{q} I_{q}, d_{1} F_{1}, d_{2} F_{2}, \cdots, d_{r} F_{r}\right)$, where $a, b_{1}, b_{2}, \cdots, b_{p}, c_{1}, c_{2}, \cdots, c_{q}, d_{1}, d_{2}, \cdots, d_{r} \in \mathbb{F}$. The set $R N Q F_{p q r}$ defined by

$$
\begin{gathered}
R N Q F_{p q r}=\left\{\left(a, b_{1} T_{1}, b_{2} T_{2}, \cdots, b_{p} T_{p}, c_{1} I_{1}, c_{2} I_{2}, \cdots, c_{q} I_{q}, d_{1} F_{1}, d_{2} F_{2}, \cdots, d_{r} F_{r}\right) \mid\right. \\
\left.a, b_{1}, b_{2}, \cdots, b_{p}, c_{1}, c_{2}, \cdots, c_{q}, d_{1}, d_{2}, \cdots, d_{r} \in \mathbb{F}\right\} .
\end{gathered}
$$

is called a refined neutrosophic quadruple set on field $\mathbb{F}$.

Definition 15. Let $x=\left(a, b_{1} T_{1}, b_{2} T_{2}, \cdots, b_{p} T_{p}, c_{1} I_{1}, c_{2} I_{2}, \cdots, c_{q} I_{q}, d_{1} F_{1}, d_{2} F_{2}, \cdots, d_{r} F_{r}\right)$, $y=\left(e, f_{1} T_{1}, f_{2} T_{2}, \cdots, f_{p} T_{p}, g_{1} I_{1}, g_{2} I_{2}, \cdots, g_{q} I_{q}, h_{1} F_{1}, h_{2} F_{2}, \cdots, h_{r} F_{r}\right) \in R N Q F_{p q r}$, then the addition operator is defined as follows:

$$
\begin{aligned}
x \oplus y=\quad & \left(a+e,\left(b_{1}+f_{1}\right) T_{1},\left(b_{2}+f_{2}\right) T_{2}, \cdots,\left(b_{p}+f_{p}\right) T_{p},\left(c_{1}+g_{1}\right) I_{1},\left(c_{2}+g_{2}\right) I_{2}, \cdots,\right. \\
& \left.\left(c_{q}+g_{q}\right) I_{q},\left(d_{1}+h_{1}\right) F_{1},\left(d_{2}+h_{2}\right) F_{2}, \cdots,\left(d_{r}+h_{r}\right) F_{r}\right) .
\end{aligned}
$$

Definition 16. Let $x=\left(a, b_{1} T_{1}, b_{2} T_{2}, \cdots, b_{p} T_{p}, c_{1} I_{1}, c_{2} I_{2}, \cdots, c_{q} I_{q}, d_{1} F_{1}, d_{2} F_{2}, \cdots, d_{r} F_{r}\right)$, $y=\left(e, f_{1} T_{1}, f_{2} T_{2}, \cdots, f_{p} T_{p}, g_{1} I_{1}, g_{2} I_{2}, \cdots, g_{q} I_{q}, h_{1} F_{1}, h_{2} F_{2}, \cdots, h_{r} F_{r}\right) \in R N Q F_{p q r} ;$ the neutrosophic expert considers the prevalence order $T_{1} \prec T_{2} \prec \cdots \prec T_{p} \prec I_{1} \prec I_{2} \prec \cdots \prec I_{q} \prec F_{1} \prec F_{2} \prec \cdots \prec F_{r}$. Then, the multiplication operation is defined as follows:

$$
\begin{gathered}
x * y=\left(a, b_{1} T_{1}, b_{2} T_{2}, \cdots, b_{p} T_{p}, c_{1} I_{1}, c_{2} I_{2}, \cdots, c_{q} I_{q}, d_{1} F_{1}, d_{2} F_{2}, \cdots, d_{r} F_{r}\right) \\
*\left(e, f_{1} T_{1}, f_{2} T_{2}, \cdots, f_{p} T_{p}, g_{1} I_{1}, g_{2} I_{2}, \cdots, g_{q} I_{q}, h_{1} F_{1}, h_{2} F_{2}, \cdots, h_{r} F_{r}\right) \\
=\left(a e,\left(a f_{1}+b_{1} e+b_{1} f_{1}\right) T_{1},\left(a f_{2}+b_{1} f_{2}+b_{2} e+b_{2} f_{1}+b_{2} f_{2}\right) T_{2},\right. \\
\left.\quad \cdots,\left(a h_{r}+b_{1} h_{r}+b_{2} h_{r}+\cdots+d_{r-1} h_{r}+d_{r} e+d_{r} f_{1}+\cdots+d_{r} h_{r}\right) F_{r}\right) .
\end{gathered}
$$

The neutrosophic expert considers the prevalence order $T_{1} \succ T_{2} \succ \cdots \succ T_{p} \succ I_{1} \succ I_{2} \succ \cdots \succ I_{q} \succ$ $F_{1} \succ F_{2} \succ \cdots \succ F_{r}$. Then,

$$
\begin{aligned}
x \star y= & \left(a, b_{1} T_{1}, b_{2} T_{2}, \cdots, b_{p} T_{p}, c_{1} I_{1}, c_{2} I_{2}, \cdots, c_{q} I_{q}, d_{1} F_{1}, d_{2} F_{2}, \cdots, d_{r} F_{r}\right) \\
& \star\left(e, f_{1} T_{1}, f_{2} T_{2}, \cdots, f_{p} T_{p}, g_{1} I_{1}, g_{2} I_{2}, \cdots, g_{q} I_{q}, h_{1} F_{1}, h_{2} F_{2}, \cdots, h_{r} F_{r}\right) \\
= & \left(a e,\left(a f_{1}+b_{1} f_{1}+\cdots+d_{r} f_{1}+b_{1} e+b_{1} f_{2}+b_{1} f_{3}+\cdots+b_{1} h_{r}\right) T_{1}\right. \\
& \left.\cdots,\left(a h_{r-1}+d_{r-1} e+d_{r-1} h_{r-1}+d_{r-1} h_{r}+d_{r} h_{r-1}\right) F_{r-1},\left(a h_{r}+d_{r} e+d_{r} h_{r}\right) F_{r}\right) .
\end{aligned}
$$

Similarly, we also have the following results.

Theorem 6. (RNQF pqr, $\oplus)$ is an abelian group.

Theorem 7. For the algebra system $\left(R N Q F_{p q r}, *\right)$ (or $\left.\left(R N Q F_{p q r}, \star\right)\right)$, for every element a $\in R N Q F_{p q r}$, there exists the neutral element neut $(a)$ and opposite element anti $(a)$, thus the algebra system $\left(R N Q F_{p q r}, *\right)$ (or $\left.\left(R N Q F_{p q r}, \star\right)\right)$ is a NETG.

Example 6. For algebra system $\left(R N Q F_{213}, *\right)$, and set $\mathbb{F}=\mathbb{R}, a=\left(1,0,2 T_{2},-3 I_{1}, 2 F_{1}, 0,-2 F_{3}\right)$, solve the neutral element and opposite elements of $a$.

According Algorithm 1 for algebra system $\left(R N F_{213}, *\right)$, firstly, we solve the neutral element and opposite elements of $a^{\prime}=\left(1,0,2 T_{2},-3 I_{1}\right)$ from Table 1 . We then solve the neutral element and opposite elements of $a^{\prime \prime}=\left(1,0,2 T_{2},-3 I_{1}, 2 F_{1}\right)$ from Table 4. Next we solve the neutral element and opposite elements of $a^{\prime \prime \prime}=\left(1,0,2 T_{2},-3 I_{1}, 2 F_{1}, 0\right)$ from Table 4 . Finally, we solve the neutral element and opposite elements of a from Table 4.

1. From Table 1 , neut $\left(a^{\prime}\right)=\left(1,0,0,-I_{1}\right)$ and anti $\left(a^{\prime}\right)=\left(1,0,-\frac{2}{3} T_{2}, c_{4} I_{1}\right)$, where $c_{4} \in \mathbb{R}$.

2. From Table 4 and combining the results of the above step: Being $2 \neq 0$ and $1+0+2+(-3)+2 \neq 0$, thus neut $\left(a^{\prime \prime}\right)=\left(1,0,0,-I_{1}, F_{1}\right)$ and anti $\left(a^{\prime \prime}\right)=\left(1,0,-\frac{2}{3} T_{2}, c_{4} I_{1}, c_{5} F_{1}\right)$, where $c_{4}+c_{5}=\frac{1}{6}$. 
3. From Table 4 and combining the results of the above step: Being $0=0$ and $1+0+2+(-3)+2+0 \neq 0$, thus neut $\left(a^{\prime \prime \prime}\right)=\left(1,0,0,-I_{2}, F_{1}, 0\right)$ and anti $\left(a^{\prime \prime \prime}\right)=\left(1,0,-\frac{2}{3} T_{2}, c_{4} I_{1}, c_{5} F_{1}, 0\right)$, where $c_{4}+c_{5}=\frac{1}{6}$.

4. From Table 4 and combining the results of the above step: Being $-2 \neq 0$ and $1+0+2+(-3)+2+$ $0+(-2)=0$, thus neut $(a)=\left(1,0,0,-I_{1}, F_{1}, 0,-F_{3}\right)$ and anti $(a)=\left(1,0,-\frac{2}{3} T_{2}, c_{4} I_{1}, c_{5} F_{1}, 0, c_{7} F_{3}\right)$, where $c_{4}+c_{5}=\frac{1}{6}, c_{7} \in \mathbb{R}$.

Example 7. For algebra system $\left(R N Q F_{213}, *\right)$, and set $\mathbb{F}=Z_{5}=\{[0],[1],[2],[3],[4]\}, a=\left([1],[0],[2] T_{2}\right.$, $\left.[2] I_{1},[2] F_{1}, 0,[3] F_{3}\right)$, solve the neutral element and opposite elements of $a$.

Similar to Example 6, according Algorithm 1 for algebra system $\left(R N F_{213}, *\right)$, firstly, we solve the neutral element and opposite elements of $a^{\prime}=\left([1],[0],[2] T_{2},[2] I_{1}\right)$ from Table 1 . We then solve the neutral element and opposite elements of $a^{\prime \prime}=\left([1],[0],[2] T_{2},[2] I_{1},[2] F_{1}\right)$ from Table 4 . Next, we solve the neutral element and opposite elements of $a^{\prime \prime \prime}=\left([1],[0],[2] T_{2},[2] I_{1},[2] F_{1},[0]\right)$ from Table 4 . Finally, we solve the neutral element and opposite elements of a from Table 4.

1. From Table 1, neut $\left(a^{\prime}\right)=\left([1],[0],[0],[4] I_{1}\right)$ and anti $\left(a^{\prime}\right)=\left([1],[0],[1] T_{2}, c_{4} I_{1}\right)$, where $c_{4} \in \mathbb{F}$.

2. From Table 4 and combining the results of the above step: Being $[2] \neq[0]$ and $[1]+[0]+[2]+[2]+$ $[2] \neq[0]$, thus neut $\left(a^{\prime \prime}\right)=\left([1],[0],[0],[4] I_{1},[1] F_{1}\right)$ and anti $\left(a^{\prime \prime}\right)=\left([1],[0],[1] T_{2}, c_{4} I_{1}, c_{5} F_{1}\right)$, where $c_{4}+c_{5}=[1]$.

3. From Table 4 and combining the results of the above step: Being $[0]=[0]$ and $[1]+[0]+[2]+[2]+[2]+$ $[0] \neq 0$, thus neut $\left(a^{\prime \prime \prime}\right)=\left([1],[0],[0],[4] I_{1},[1] F_{1},[0]\right)$ and anti $\left(a^{\prime \prime \prime}\right)=\left([1],[0],[1] T_{2}, c_{4} I_{1}, c_{5} F_{1},[0]\right)$, where $c_{4}+c_{5}=[1]$.

4. From Table 4 and combining the results of the above step: Being $[3] \neq[0]$ and $[1]+[0]+[2]+$ $[2]+[2]+[0]+[3]=0$, thus neut $(a)=\left([1],[0],[0],[4] I_{1},[1] F_{1},[0],[4] F_{3}\right)$ and anti $(a)=$ $\left([1],[0],[1] T_{2}, c_{4} I_{1}, c_{5} F_{1},[0], c_{7} F_{3}\right)$, where $c_{4}+c_{5}=[1], c_{7} \in \mathbb{F}$.

\section{Conclusions}

In this paper, we study the algebra structure of $\left(N Q F\left(R N F_{n}, R N Q F_{n}\right), \oplus\right),\left(N Q F\left(R N F_{n}, R N Q F_{n}\right), *\right)$ and $\left(N Q F\left(R N F_{n}, R N Q F_{n}\right), \star\right)$, and we prove that $\left(N Q F\left(R N F_{n}, R N Q F_{n}\right), *\right)$ (or $\left(N Q F\left(R N F_{n}\right.\right.$, $\left.\left.R N Q F_{n}\right), \star\right)$ ) is a neutrosophic extended triplet group, and provide new examples of neutrosophic extended triplet group and the neutral element and opposite elements of each refined $n$-ary neutrosophic number (refined neutrosophic quadruple number) can be obtained by given algorithms. In the following, we can explore the algebra structure of $\left(N Q F\left(R N F_{n}, R N Q F_{n}\right), \oplus, *\right)$ or $\left(N Q F\left(R N F_{n}, R N Q F_{n}\right), \oplus, \star\right)$. We can also explore the relation of neutrosophic quadruple numbers and other algebra systems in papers [21-23]. Moreover, on the one hand, we will discuss the neutrosophic quadruple numbers based on some particular ring which can form a neutrosophic extended triplet group, while, on the other hand, we will introduce a new operation $\circ$ in order to guarantee $\left(N Q F\left(R N F_{n}, R N Q F_{n}\right), *, 0\right)$ is a neutrosophic triplet ring.

Author Contributions: All authors contributed equally to this paper.

Funding: This research was funded by National Natural Science Foundation of China (Grant No. 11501435), Discipline Construction Funding of Xi'an Polytechnic University, Instructional Science and Technology Plan Projects of China National Textile and Apparel Council (No. 2016073) and Scientific Research Program Funded by Shaanxi Provincial Education Department (Program No. 18JS042).

Acknowledgments: The authors would like to thank the reviewers for their many insightful comments and suggestions.

Conflicts of Interest: The authors declare no conflicts of interest.

\section{References}

1. Smarandache, F. A Unifying Field in Logics. Neutrosophy: Neutrosophic Probability, Set and Logic; American Research Press: Rehoboth, TX, USA, 1998.

2. Smarandache, F.; Ali, M. Neutrosophic triplet group. Neural Comput. Appl. 2018, 29, 595-601. [CrossRef] 
3. Smarandache, F. Neutrosophic Perspectives: Triplets, Duplets, Multisets, Hybrid Operators, Modal Logic, Hedge Algebras. And Applications; Pons Publishing House: Brussels, Belgium, 2017.

4. Zhang, X.; Hu, Q.; Smarandache, F.; An, X. On Neutrosophic Triplet Groups: Basic Properties, NT-Subgroups, and Some Notes. Symmetry 2018, 10, 289. [CrossRef]

5. Zhang, X.H.; Bo, C.X.; Smarandache, F.; Dai, J.H. New inclusion relation of neutrosophic sets with applications and related lattice structure. Int. J. Mach. Learn. Cybern. 2018, 9, 1753-1763. [CrossRef]

6. Bal, M.; Shalla, M.M.; Olgun, N. Neutrosophic Triplet Cosets and Quotient Groups. Symmetry 2017, 10, 126. [CrossRef]

7. Zhang, X.H.; Smarandache, F.; Liang, X.L. Neutrosophic Duplet Semi-Group and Cancellable Neutrosophic Triplet Groups. Symmetry 2017, 9, 275. [CrossRef]

8. Ma, Y.; Zhang, X.; Yang, X.; Zhou, X. Generalized Neutrosophic Extended Triplet Group. Symmetry 2019, 11, 327. [CrossRef]

9. Wu, X.Y.; Zhang, X.H. The decomposition theorems of AG-neutrosophic extended triplet loops and strong AG-(1, 1)-loops. Mathematics 2019, 7, 268, doi:10.3390/math7030268. [CrossRef]

10. Zhang, X.H.; Wu, X.Y.; Mao, X.Y.; Smarandache, F.; Park, C. On Neutrosophic Extended Triplet Groups (Loops) and Abel-Grassmann's Groupoids (AG-Groupoids). J. Intell. Fuzzy Syst. 2019, in press.

11. Zhang, X.H.; Mao, X.Y.; Wu, Y.T.; Zhai, X.H. Neutrosophic filters in pseudo-BCI algebras. Int. J. Uncertain. Quant. 2018, 8, 511-526. [CrossRef]

12. Smarandache, F. Hybrid Neutrosophic Triplet Ring in Physical Structures. Bull. Am. Phys. Soc. 2017, 62, 17.

13. Ali, M.; Smarandache, F.; Khan, M. Study on the development of neutrosophictriplet ring and neutrosophictriplet field. Mathematics 2018, 6, 46. [CrossRef]

14. Sahin, M.; Abdullah, K. Neutrosophic triplet normed space. Open Phys. 2017, 15, 697-704. [CrossRef]

15. Zhang, X.H.; Bo, C.X.; Smarandache, F.; Park, C. New operations of totally dependent-neutrosophic sets and totally dependent-neutrosophic soft sets. Symmetry 2018, 10, 187. [CrossRef]

16. Agboola, A.; Akinleye, S. Neutrosophic Vector Spaces. Neutrosophic Sets Syst. 2014, 4, 9-18.

17. Li, Q.; Ma, Y.; Zhang, X.; Zhang, J. Neutrosophic Extended Triplet Group Based on Neutrosophic Quadruple Numbers. Symmetry 2019, 11, 187. [CrossRef]

18. Smarandache, F. Neutrosophic Quadruple Numbers, Refined Neutrosophic Quadruple Numbers, Absorbance Law, and the Multiplication of Neutrosophic Quadruple Numbers. Neutrosophic Sets Syst. 2015, 10, 96-98.

19. Smarandache, F. (T, I, F)-Neutrosophic Structures. Neutrosophic Sets Syst. 2015, 8, 3-10. [CrossRef]

20. Akinleye1, S.A.; Smarandache, F.; Agboola, A.A.A. On Neutrosophic Quadruple Algebraic Structures. Neutrosophic Sets Syst. 2016, 12, 122-126.

21. Zhang, X.H. Fuzzy anti-grouped filters and fuzzy normal filters in pseudo-BCI algebras. J. Intell. Fuzzy Syst. 2017, 33, 1767-1774. [CrossRef]

22. Zhang, X.H.; Borzooei, R.A.; Jun, Y.B. Q-filters of quantum B-algebras and basic implication algebras. Symmetry 2018, 10, 573, doi.10.3390/sym10110573. [CrossRef]

23. Zhang, X.H.; Park, C.; Wu, S.P. Soft set theoretical approach to pseudo-BCI algebras. J. Intell. Fuzzy Syst. 2018, 34, 559-568. [CrossRef]

(C) 2019 by the authors. Licensee MDPI, Basel, Switzerland. This article is an open access article distributed under the terms and conditions of the Creative Commons Attribution (CC BY) license (http://creativecommons.org/licenses/by/4.0/). 